\title{
ETHINYL ESTADIOL/PROGESTIN ORAL CONTRACEPTIVES DEPRESS SPATIAL LEARNING AND DYSREGULATE HIPPOCAMPAL CA3 MICROSTRUCTURE: IMPLICATIONS FOR BEHAVIORAL-COGNITIVE EFFECTS OF CHRONIC CONTRACEPTIVE USE?
}

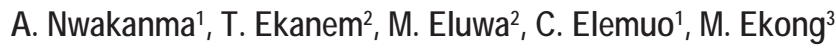 \\ ${ }^{1}$ Department of Anatomy, Chukwuemeka Odumegwu Ojukwu University, Uli - Nigeria \\ ${ }^{2}$ Department of Anatomy, University of Calabar, Calabar - Nigeria \\ ${ }^{3}$ Department of Anatomy, University of Uyo, Uyo - Nigeria
}

\begin{abstract}
Combined oral contraceptive pill contains ethinyl estradiol and a synthetic progestin, which prevent ovulation by suppressing the release of the gonadotropins resulting in the inhibition of ovarian follicles' development. Although advantageous in birth control, the impact on learning and memory is limited necessitating this study on its effect on spatial learning, and hippocampal CA3 microstructure. Thirty two female Wistar rats of average body weight $200 \mathrm{~g}$ were equally divided $(n=8)$ into four groups; $0.002 \mathrm{mg} / \mathrm{kg}$ levonorgestrel plus $0.00043 \mathrm{mg} / \mathrm{kg}$ ethinyl estradiol (COCP) were administered orally for 21, 42 and 63 days. 24 hours after the last administration the rats underwent Morris water maze test and were sacrificed by transcardial perfusion-fixation. Their hippocampal regions were processed for histological study, and immunolabelled with anti-neuron specific enolase (NSE) and glial fibrillary acidic protein (GFAP). Results showed that the COCP test groups had shorter escape latencies $(p \leq 0.05)$ in the visible and hidden platform trials. The COCP test groups showed no difference in neuronal population, although some of the hippocampal CA3 pyramidal neurons were either atrophic and/or karyorrhectic, with shrunken and dense nuclei. NSE expression was lower $(p \leq 0.05)$ in the 21, 42 and 63 days COCP groups, while GFAP expression was lower in the 21 days COCP group, but not different in the 42 and 63 days COCP groups compared with the control. These preliminary results show that COCP influence spatial learning, and may also reduce neuronal metabolic activity, while increasing astrocytic activity in the hippocampal CA3.
\end{abstract}

Key words: combined oral contraceptive pill; Morris water maze; histomorphology; immunohistochemistry; hippocampus; Wistar rats

Corresponding author: A.A. Nwakanma, Department of Anatomy, Chukwuemeka Odumegwu Ojukwu University, Uli, Nigeria, Phone: +2348037738053, e-mail: akudoekeoma@yahoo.com

LAST REVISED: 20 January 2021 ACCEPTED: 26 January 2021 


\section{INTRODUCTION}

$\mathrm{B}$ irth control is a major factor in public health and welfare, and this preserves the general and reproductive health of women by allowing for planned pregnancy [1]. One method of planned pregnancy is the use of the oral contraceptive pills, in which earlier prescriptions contained only ethinyl estradiol or synthetic progestin, and often called the „minipill“ [2]. Nowadays, combined oral contraceptives contain these two hormones [3]. Ethinyl estradiol is very stable but is usually metabolised in the liver to 2-hydroxy ethinyl estradiol, while progestin is also metabolized to hydroxypregnanolone or pregnanediol [4]. Many current oral contraceptive combined brands use lower ethinyl estradiol concentrations than previously, and are proving to be safer while still being effective $[5,6]$.

The combined oral contraceptive pill primarily prevents ovulation by inhibiting follicular development and consequently, corpus luteum formation. This ovarian inhibitory action which is effective at the hypothalamus, targets the pituitary production and secretion of the gonadotropins $[7,8]$. It is reported that almost two million women worldwide use oral contraception [9], with beneficial effects of reducing the incidence of pelvic inflammatory disease, decreasing risk of ectopic pregnancy, benign breast lesions, ovarian and endometrial cancers, and protecting against osteoporosis and rheumatoid arthritis among the users $[6,10,11]$. In addition to these hormonal effects on reproductive function, they also exhibit modulatory effects on neurochemical systems involved in emotional and cognitive functions, that is, noradrenergic, dopaminergic, serotoninergic, glutamatergic and GABAergic systems [12].

Oral contraceptives have different reported roles in cognitive abilities and emotional recognition [13, 14]. However, in ovariectomized animals, ethinyl estradiol showed conflicting results; had no effect on spatial and object memory $[15,16]$ or impaired acquisition of spatial navigation skills and strategies [17]. Synthetic progestin on the other hand either had no effect on water maze activity [18] or enhanced working memory [19], while its metabolite, allopregnanolone inhibited learning in the Morris water maze [20]. But despite their well-characterized effects, little is known about the combined oral contraceptives mechanisms of action on cognitive abilities [13, 14, 21]. Knowledge about the impact of combined oral contraceptives on cognitive abilities is of great importance equally to the private user and physicians, as well as to clinical and fundamental researches. Cognitive function is, however, a broad concept and includes a lot of different capacities. A research report suggests that the synthetic steroids delivered by the female contraceptive pill can shrink certain regions of the female brain and could also alter their functions [22]. Thus, the hippocampus forms the integral part of the present study.

The hippocampus is a very important brain region with diverse cognitive abilities, and especially in learning and memory. It is a C-shaped structure also called the cornu ammoni and located deep in the temporal lobe [23]. The CA3 region is reported to decrease in metabolic activity following fear conditioned learning [24], which at the same time cause decreased water maze activity, attributed to the CA3 role in the storage of water maze representations [25]. Nevertheless, there is paucity of information on how oral contraceptives affect learning and memory, and the structure of the hippocampus, especially the CA3 region. This limited information and the need to assess learning and memory motivated this investigation on the role of combined oral contraceptives on hippocampal CA3 structure and function.

\section{MATERIALS AND METHODS}

Thirty two female Wistar rats of average body weight $200 \mathrm{~g}$ were equally divided $(n=8)$ into four groups. The animals were handled according to the guidelines for animal care of the United States National Institute of Health, and they were allowed access to rat chow (Vital Feeds Limited, Nigeria) and water ad libitum throughout the experimental period.

Three packets of Combination3 $₫$ were obtained from a reputable Pharmacy in Owerri, Nigeria, with each packet containing 21 beige-coloured tablets made up of $0.15 \mathrm{mg}$ levonorgestrel and $0.03 \mathrm{mg}$ ethinyl estradiol, and 7 brown coloured tablets containing 75 $\mathrm{mg}$ ferrous fumarate. Each tablet was grounded to powder and dissolved in $175 \mathrm{ml}$ of distilled water. The therapeutic dosages were determined per body weight of the animals using $60 \mathrm{~kg}$ body weight for mature human females. The preparation was carried out daily before administration.

Oral contraceptives produce a cycle of serum levels throughout the day, with concentrations as high as 1-2 hours after ingestion of the pill [26]. The four groups were the control group (group 1 - untreated), and test groups (groups 2, 3 and 4). $0.002 \mathrm{mg} / \mathrm{kg}$ levonorgestrel plus $0.00043 \mathrm{mg} / \mathrm{kg}$ ethinyl estradiol (COCP) were administered orally, for 21,42 and 63 days, respectively (Table 1). Twenty four hours after the last administrations for each group, behavioural task in the Morris water maze (MWM) was carried out [27]. The three groups who received levonorgestrel plus ethinyl estradiol tested on the Morris water maze on days 22- 
$27,43-48$ and 64-69. The control group rats were also exposed to the test on days 64-69 of the experiment.

Briefly, a black circular pool with diameter $150 \mathrm{~cm}$ and depth $50 \mathrm{~cm}$ was kept in a neurobehaviour room with contrast spatial cues placed on the walls. A $10 \mathrm{~cm}$ diameter platform was placed in the pool. The pool was then filled with water until the platform was a centimetre above the water surface. The water was left overnight in the neurobehaviour room to attain room temperature $\left(27-29^{\circ} \mathrm{C}\right)$. The training consisted of a day of cue (visible) platform training (four trials per day) followed immediately by 4 days of place (hidden) platform training (four trials per day). Each rat was given 60 seconds to locate the platform and was allowed to remain on the platform for 5 seconds and then returned to a holding cage. The time to discovered and mount the platform (escape latency) was noted as index of retrieval of spatial learning [27, 28].

The rats were then anaesthetised with $40 \mathrm{mg} / \mathrm{kg}$ ketamine (i.p.), and the animals perfused transcardially with $1 \mathrm{M}$ phosphate buffered saline and subsequently with $10 \%$ neutral buffered formalin (NBF). Each rat's whole brain was excised and post-fixed in $10 \%$ NBF for 48 hours. The brain tissue at the level of the hippocampus was excised and routinely processed for paraffin sectioning. Serial representative sections were stained with haematoxylin and eosin ( $H$ \& E), and Cresyl fast violet (CFV), while others were immnunolabelled with anti-neuron specific enolase (NSE) and glial fibrillary acidic protein (GFAP) after antigen retrieval.

Briefly, serial paraffin sections on slides were brought to water and antigen retrieval was performed using citrate buffer ( $\mathrm{pH} \mathrm{6.0)}$ in a microwave oven for 5 minutes, followed by protein block using $3 \%$ hydrogen peroxide for 10 minutes. Sections were thereafter preincubated in $2 \%$ normal goat serum for 30 minutes and incubated in monoclonal mouse anti-enolase-2 (Novocastra, Leica Biosystems, 22C9, 1:100) for NSE, and mouse monoclonal anti GFAP (Novocastra, Leica Biosystems, NCL-L-GFAP-GA5, 1:100) for GFAP, followed by 1 hour incubation in goat anti-mouse secondary antibody (1:100) for an hour. Detection of reaction was by means of the avidin-biotin complex with diaminobenzidine as the chromagen. Sections were then counterstained with Harris haematoxylin, dehydrated, cleared and cover-slipped with DPX. Processed slides were viewed under the light microscope and photomicrographs obtained using a computer assisted digital microscope's camera.
Cellular density was determined manually by means of Image $囚$ software (version 1.37c). Briefly, images of the hippocampal CA3 region was obtained for each section and randomly mapped with the Image ${ }^{\circledR}$ gridlines. Counting of cell nuclei was done manually taking into consideration the nuclei on the upper and right borders of the mapped areas.

Data were analyzed using repeated measure analysis of variance for the behavioural parameters and one way analysis of variance for cell count; by means of Graphpad prism (version 5) and post-hoc Tukey test used to compare group difference. Statistically significant data were determined at probability level $p \leq 0.05$, and data represented as mean \pm standard error of mean.

Table 1. Schedule of Drug Administration

\begin{tabular}{|l|l|l|}
\hline Groups $(\mathbf{n}=8)$ & Dosage of Combination 3 & Duration \\
\hline 1 (control ) & No treatment & 63 days \\
\hline 2 & $\begin{array}{l}0.002 \mathrm{mg} / \mathrm{kg} \text { levonorgestrel plus } \\
0.00043 \mathrm{mg} / \mathrm{kg} \text { ethinyl estradiol }\end{array}$ & 21 days \\
\hline 3 & $0.002 \mathrm{mg} / \mathrm{kg}$ levonorgestrel plus & \multirow{2}{*}{42 days } \\
\hline 4 & $0.00043 \mathrm{mg} / \mathrm{kg}$ ethinyl estradiol & \\
\hline & $0.002 \mathrm{mg} / \mathrm{kg}$ levonorgestrel plus & 63 days \\
\hline
\end{tabular}

\section{RESULTS}

\section{Neurobehavioural study}

Escape latency in Morris water maze behavioural study: On trial day 1 (the visible platform trial), test groups administered COCP for 21, 42 and 63 days had significantly $(p \leq 0.05)$ shorter escape latencies compared with the control group. On trial days 2-5 (the hidden platform trial), the 21, 42 and 63 days COCP groups also had significantly $(p \leq 0.05)$ shorter escape latencies on days 4 and 5 , while the 21 days COCP group had a significantly ( $p \leq 0.05)$ shorter escape latency on day 3 compared with the control group (Figure 1).

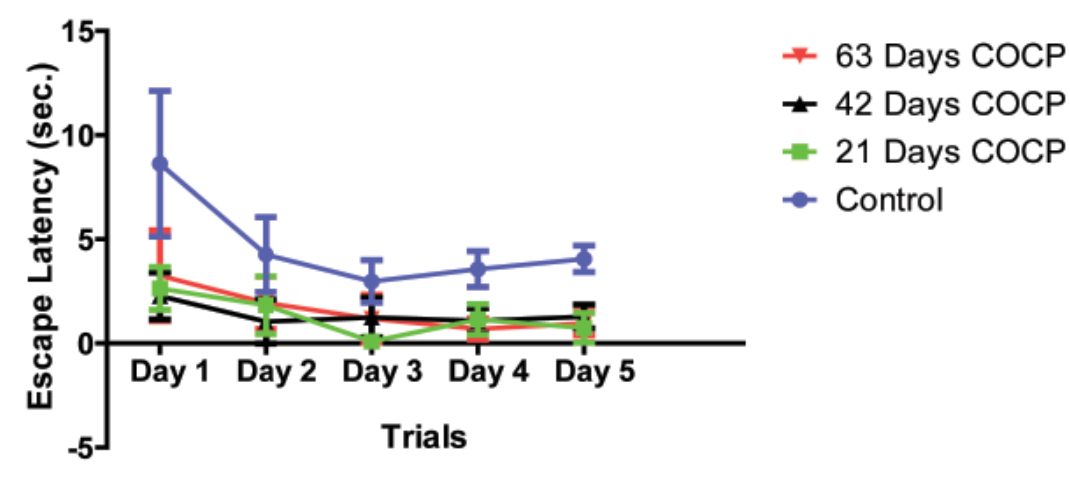

Results are presented as Mean \pm Standard error of mean $n=8$ COCP - Combined oral contraceptive pill

Fig. 1. Escape latency in the Morris water maze of the COCP and control groups 


\section{Histomorphology study}

The H \& E stained histomorphology of the hippocampal CA3 of the control group showed three layers, from the outside inwards; molecular, pyramidal and polymorphic. The molecular layer showed sparsely distributed cells, while the pyramidal layer had a large and dense population of pyramidal cells. The polymorphic layer also showed sparse cell distribution (Figure 2a).

The histomorphology of the hippocampal CA3 of the 21 days COCP group showed large pyramidal neurons having some karyorrhectic nuclei compared with the control group (Figure 2b). The histomorphology of the hippocampal CA3 of the 42 days COCP group showed slight atrophy and karyorrhexis of some of the pyramidal neurons compared with the control group (Figure 2c). The histomorphology of the hippocampal CA3 of the 63 days COCP group also showed slight atrophy and karyorrhexis of some of the pyramidal neurons compared with the control group (Figure 2d). Hippocampal CA3 cellular population was not different $(p>0.05)$ between the COCP test groups and the control group (Figure 2e).

The CFV stained histomorphology of the hippocampal CA3 of the control group showed prominent large pyramidal (Py) neurons having normal histomorphology (Figure 3a). The hippocampal CA3 of the COCP group for 21 days showed pyramidal neurons with shrunken and dense soma compared with the control group (Figure 3b). The hippocampal CA3 of the COCP group for 42 days showed pyramidal neurons with shrunken and dense soma compared with the control group (Figure 3c). The hippocampal CA3 of the COCP group for 63 days also showed pyramidal neurons with shrunken and dense soma compared with the control group (Figure 3d). Hippocampal CA3 neuronal population was not different $(p>0.05)$ between the COCP test groups and the control group (Figure $3 \mathrm{e}$ ).

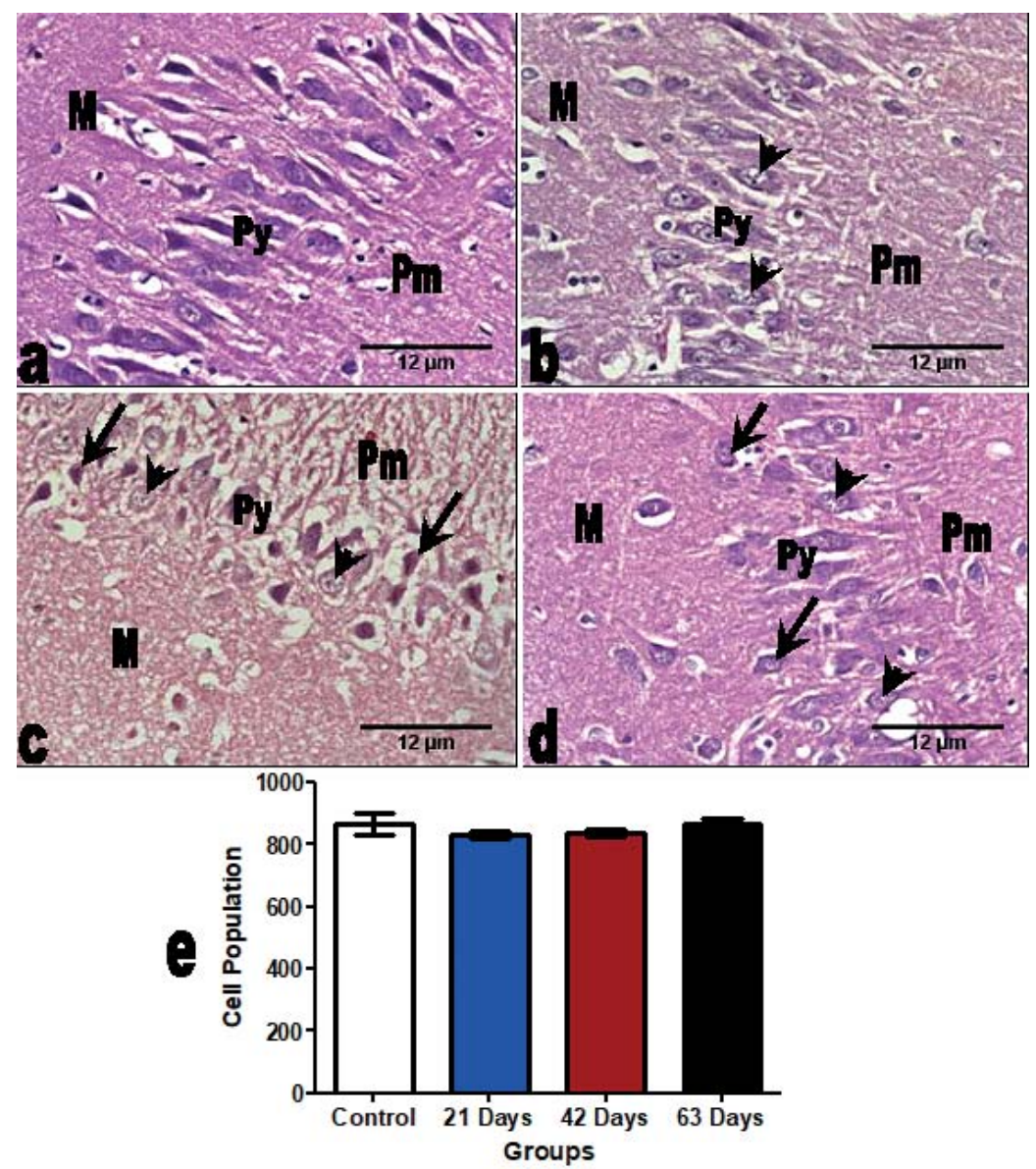

Fig. 2. The sections of the hippocampal CA3 and their cellular population in the control and the COCP groups administered for 21,42 and 63 days $(\mathrm{H} \& \mathrm{E}, \times 400)$ :

a. The hippocampal CA3 of the control group showing three layers; molecular (M), pyramidal (Py) and polymorphic (Pm) with normal histomorphology.

b. There were large pyramidal neurons with some karyorrhectic nuclei (arrow head) in the 21 days COCP group.

c. There were slight atrophy (arrow) and karyorrhexis (arrow head) of some of the pyramidal neurons in the 42 days COCP group.

d. There were slight atrophy (arrow) and karyorrhexis (arrow head) of some of the pyramidal neurons in the 63 days COCP group.

e. No significant difference $(p>0.05)$ among groups 


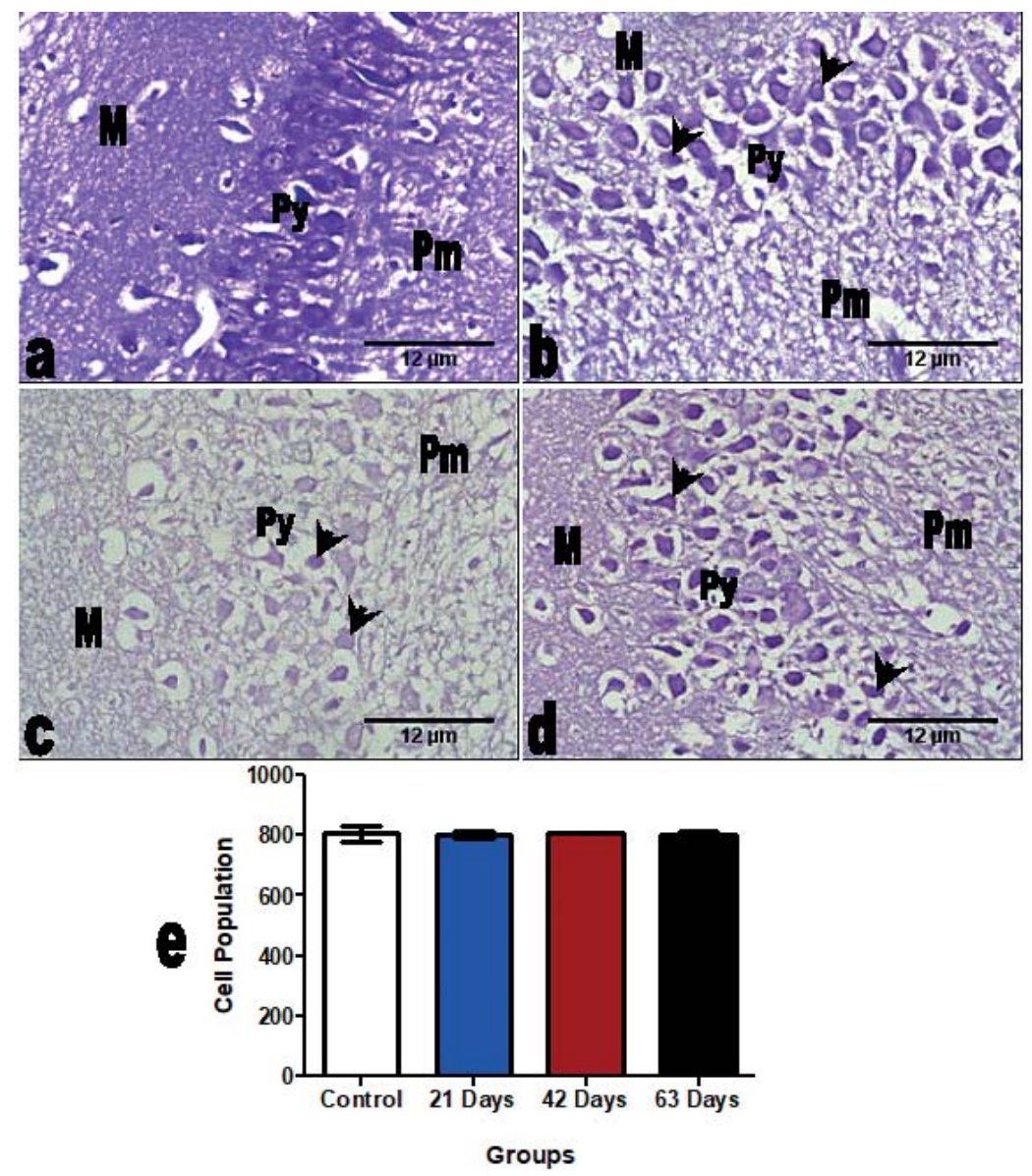

Fig. 3. The sections of the hippocampal CA3 and their cellular population in the control and the COCP groups administered for 21,42 and 63 days $(\mathrm{CFV}, \times 400)$ :

a. The hippocampal CA3 of the control group showing prominent large pyramidal (Py) neurons having normal histomorphology.

b. The hippocampal CA3 pyramidal neurons appear shrunken with dense nuclei (arrow head) in the 21 days COCP group.

c. The hippocampal CA3 pyramidal neurons appear shrunken with dense nuclei (arrow head) in the 42 days COCP group.

d. The hippocampal CA3 pyramidal neurons appear shrunken with dense nuclei (arrow head) in the 63 days COCP group.

e. No significant difference $(p>0.05)$ among group

\section{Immunolabelling study}

There was NSE expression in the hippocampal CA3 pyramidal neurons of the control group (Figure 4a). NSE expression was decreased in the pyramidal neurons of CA3 in the 21 days COCP group compared with the control group (Figure 4b). NSE expression was decreased in the pyramidal neurons of CA3 in the 42 days COCP group compared with the control group (Figure 4c). NSE expression was also decreased in the pyramidal neuron in the 63 days COCP group compared with the control group (Figure $4 d$ ).

NSE labelled cells were significantly lower $(p \leq 0.05)$ in the 21, 42 and 63 days COCP groups compared with the control group. There was no difference $(p$ $>0.05)$ in the NSE labelled cells among the COCP groups (Figure 4e).

There was GFAP expression in the hippocampal CA3 of the control group especially in the processes
(Figure 5a). There was increased GFAP expression especially in the soma and processes throughout the CA3 layers of the 21 days COCP group compared with the control group (Figure 5b). GFAP expression was increased in the soma and processes in the CA3 layers of the 42 days COCP group compared with the control group (Figure 5c), while GFAP expression was also increased in the soma and processes of the CA3 layers of the 63 days COCP group compared with the control group (Figure $5 d$ ).

GFAP labelled astrocytes were significantly lower ( $p \leq$ $0.05)$ in the 21 days COCP group, but not different ( $p$ $>0.05$ ) in the 42 and 63 days COCP group compared with the control group. GFAP labelled astrocytes in the 21 days COCP group were significantly lower ( $p$ $\leq 0.05$ ) than the 42 and 63 days COCP groups. However, there was no difference $(p>0.05)$ in the GFAP labelled astrocyte between the 63 days COCP group and the 42 days COCP group (Figure $5 \mathrm{e}$ ). 

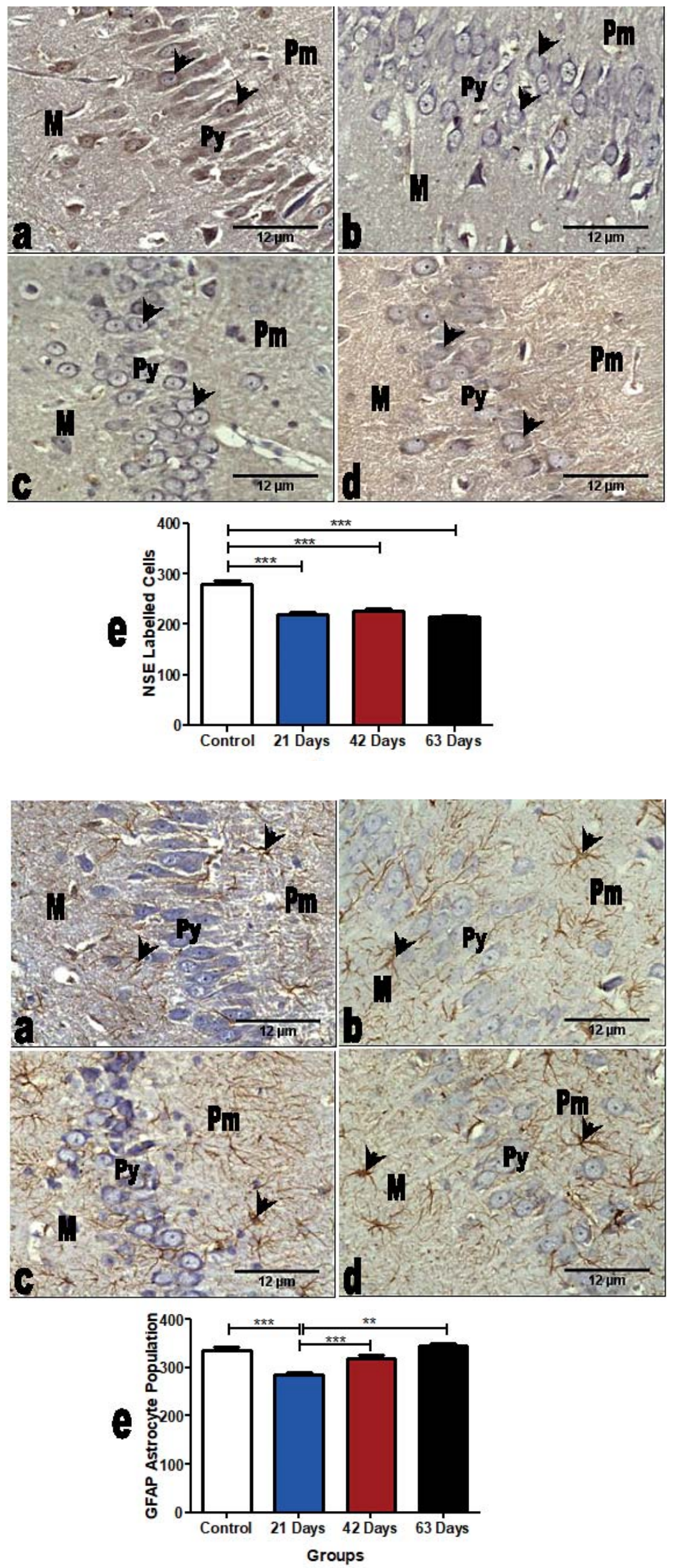

Fig. 4. The sections of the hippocampal CA3 and their NSE labelled population in the control and the COCP groups administered for 21, 42 and 63 days (NSE, $\times 400$ ):

a. There was NSE expression in the pyramidal neurons (arrow head) in the control group.

b. There was decreased NSE expression in the pyramidal neurons (arrow head) in the 21 days COCP group.

c. There was decreased NSE expression in the pyramidal neuron (arrow head) in the 42 days COCP group.

d. There was slight decreased NSE expression in the pyramidal neuron (arrow head) in the 63 days COCP group.

e. ${ }^{* *}$ Significantly different at $p \leq 0.001$ with the control group

Fig. 5. The sections of the hippocampal CA3 and the GFAP labelled cellular population in the control and the COCP groups administered for 21, 42 and 63 days (GFAP, $\times 400$ ):

a. There was GFAP expression (arrow head) in the control group.

b. There was increased GFAP expression with more prominent soma and processes (arrow head) in the 21 days COCP group.

c. There was increased GFAP expression with more prominent soma and processes (arrow head) in the 42 days COCP group.

d. There was decreased GFAP expression with more prominent soma and processes (arrow head) in the 63 days COCP group.

e. ${ }^{* *},{ }^{* *}$ Significantly different at $p \leq 0.01$ and 0.001 , respectively, with the control group 


\section{DISCUSSION}

The present study assessed spatial learning and the accompanying histomorphology of the CA3 brain area after combined oral contraceptive administration. In the MWM task, all the COCP test groups had shorter escape latencies in the visible and hidden platform trials. The MWM task is used to assess learning and memory in animals, and these animals utilize visual stimuli from extra-maze cues in the test room to form spatial orientation map in the brain [27, $28,29]$. The COCP test group could be said to utilize their visual cues more effectively or that the control group animals swarm slower than the COCP groups, which may be a reason for the less escape latency. But similar dose of ethinyl estradiol alone in ovariectomized rats did not impact working memory, but transiently impaired reference memory during the learning phase of testing [16]. In the case of levonorgestrel alone, it enhanced working memory in ovariectomized animals [19]. The difference may be due to limitation of the endogenous ovarian hormones' influence as in post-menopausal individuals in the reported results, and also may be due to the combined actions of the two hormones in the COCP groups of the present study.

Oral contraceptives have been reported to have physiological effects on areas of the brain that are associated with higher-order cognitive tasks and emotional brain reactivity [13,30], and contraceptive users have been reported to perform better on tasks of reaction time, short-term memory, sustained attention and emotional memory recall [8, 21, 31, 32]. The present results suggest that continual usage of COCP may not be advantageous for learning as the two hormones actions may be antagonistic.

In the histomorphology studies, some of the hippocampal CA3 pyramidal neurons of the COCP test groups were atrophic and/or karyorrhectic with no difference in cell population compared with the control group. The morphological changes observed in this study indicate that COCP may lead to stress or trauma to this brain area, irrespective of the duration of administration. COCP is reported to cause stress and also influence the release of stress hormones [21, 33]. There are reports that hippocampal CA3 pyramidal neurons and those of the amygdala are affected by repeated restraint stress or a combination of daily stressors resulting in atrophy or remodelling of hippocampal CA3 pyramidal neurons $[34,35,36]$. Transient reduction in the number of dendritic spines and branches of pyramidal neurons in the CA3 was also reported when the hippocampus is stressed [37], which may also be a reason for the result of the present study.
The Cresyl violet stained hippocampal CA3 of all the COCP test groups showed shrunken and dense pyramidal neurons soma with no difference in cell population compared with the control group, indicating trauma to the pyramidal neurons with the COCP administration. This supports the previous results of the $\mathrm{H}$ and $\mathrm{E}$ sections in the present study.

NSE expression was decreased in the pyramidal neurons of CA3 in all the COCP test groups, and these neuronal expressions were significantly lower in the 21, 42 and 63 days COCP groups compared with the control group. NSE is a glycolytic enzyme that serves as a neuronal marker and as marker of neuronal functional metabolic activity [38, 39]. This enzyme has a neurotrophic and neuroprotective effects on neurons in the CNS mediated by specific binding to the neuronal surface [40], and serves as a good indicator of neuronal damage. Decreased NSE expression may indicate degradation of this cytosolic enzyme [41] in response to stress, with a resulting reduction in capacity for neuronal metabolism. It is reported that continuous stressors to the brain may result in decrease metabolic activity in the hippocampus [42, 43]. Also, decreased NSE expressed cells may be an indicator of neuronal damage independent of stress response. It is reported that ethinyl estradiol reduced the number of cholinergic cells in the basal forebrain [16], and this may have affected the hippocampus also, as it is the site for estrogen receptors, whose neurons project from the basal forebrain cells [44].

GFAP expression increased in the soma and processes of the CA3 layers of all the COCP test groups, and these expressed astrocytes were significantly lower in the 21 days COCP group, but not different in the 42 and 63 days COCP group compared with the control group. Increased GFAP expression has been associated with the up-regulation of the GFAP protein in astrocytes, which usually arise following injury or trauma to the central nervous system [45]. Thus, increased GFAP expression is essential for the process of reactive astrogliosis [46. 47], but this may be either physiological or pathological. As a physiological process, it may be protective to the neurons from the stress of the COCP which has been reported to cause hippocampal CA3 pyramidal atrophy and remodelling [21, 48], and supports the NSE decreased expression, vis its energy metabolism. However, if pathological, may result in a cascade of processes leading to degeneration [45]. Therefore, the increased GFAP expressions in the present study may be protective, but continuous stress on this brain area may lead to irreparable consequences. 
The hippocampal CA3 is the brain area responsible for emotional learning and memory consolidation [23], and an important brain target for glucocorticoids, because of its high concentrations of glucocorticoid and other hormones receptors [7, 8, 49]. Its pyramidal cells are among the first to be affected in a variety of conditions that lead to loss of memory and intellectual functions as well as morphological alternations $[34,49,50]$.

\section{CONCLUSION}

These preliminary results show that COCP influenced learning, and may reduce neuronal metabolic activity, while also increasing astrocytic activity in the hippocampal CA3. Although COCP action is not duration dependent, it may be important clinically for the understanding of the influence of sex hormones on disorders related to spatial learning.

\section{REFERENCES}

1. Kapp N. WHO provider brief on hormonal contraception and liver disease. Contraception. 2009; 80:325-326.

2. Schwartz JL, Gabelnick HL. Current contraceptive research. Perspect Sexualm Reprod Health. 2002; 34(6):310-316.

3. Stanczyk FZ, Archer DF, Bhavnani BR. Ethinyl estradiol and $17 \beta$-estradiol in combined oral contraceptives: pharmacokinetics, pharmacodynamics and risk assessment. Contraception. 2013; 87(6):706-727.

4. Zhang K, Fent K. Determination of two progestin metabolites (17 $\alpha$ hydroxypregnanolone and pregnanediol) and different classes of steroids (androgens, estrogens, corticosteroids, progestins) in rivers and waste waters by high-performance liquid chromatography tandem mass spectrometry (HPLCMS/MS). Sci Total Environ. 2018; 610-611:1164-1172.

5. Golobof A, Kiley J. The current status of oral contraceptives: progress and recent innovations. Semin Reprod Med. 2016; 34(3):145-151.

6. Dikke GB. Protective potential and safety of multiphase combined oralcontraception based on natural estrogen in mature women. Problems Reprod. 2018; 23(3):27-33.

7. Rivera R, Yacobson I, Grimes D. The mechanism of action of hormonal contraceptives and intrauterine contraceptive devices. Am J Obstet Gynecol. 1999; 181(5):1263-1269.

8. Nelson AL, Cwiak C. Combined oral contraceptives. In: Hatcher RA, Trussel J, Nelson A, et al. (Eds.). Contraceptive Technology. 20th ed. New York, Ardent Media. 2011, 249-342.

9. United Nations. Population growth and universal access to reproductive health. Report of the United Nations Population Division. Department of Economic and Social Affairs. 2014. http:// www.un.org/en/development/desa/population/publications/pdf/ popfacts/Pop Facts_2014-6.pdf. Accessed on June 24, 2017.

10. De Leo V, Musacchio MC, Cappelli V, et al. Hormonal contraceptives: pharmacology tailored to women's health. Human Reprod Update. 2016; 22(5):634-646.

11. Michels KA, Brinton LA, Pfeiffer RM, et al. Oral contraceptive use and risks of cancer in the NIH-AARP diet and health study. Am J Epidemiol. 2018; 187(8):1630-1641.

12. Toffoletto $S$, Lanzenberger $R$, Gingnell $M$, et al. Emotional and cognitive functional imaging of estrogen and progester- one effects in the female human brain: a systematic review. Psychoneuroendocrinol. 2014; 50:28-52.

13. Gingnell M, Engman J, Frick A, et al. Oral contraceptive use changes brain activity and mood in women with previous negative effect on the pill- A double-blinded, placebo-controlled randomized trial of a levonorgestrel-containing combined oral contraceptive. Psychoneuroendocrinol, 2013; 38(7):1133-1144.

14. Pletzer B, Kronbichler M, Kerschbaum H. Differential effects of androgenic and anti-androgenic progestins on fusiform and frontal gray matter volume and face recognition performance. Brain Res. 2015; 1596:108-115.

15. Gresack JE, Frick KM. Effects of continuous and intermittent estrogen treatments on memory in aging female mice. Brain Res. 2006; 1115:135-147.

16. Mennenga SE, Gerson JE, Koebele SV, et al. Understanding the cognitive impact of the contraceptive estrogen ethinyl estradiol: tonic and cyclic administration impairs memory, and performance correlates with basal forebrain cholinergic system integrity. Psychoneuroendocrinol. 2015; 54:1-13.

17. Snihur AW, Hampson E, Cain DP. Estradiol and corticosterone independently impair spatial navigation in the Morris water maze in adult female rats. Behav Brain Res. 2008; 187(1):56-66.

18. Frye CA, Walf AA. Progesterone to ovariectomized mice enhances cognitive performance in the spontaneous alternation, object recognition, but not placement, water maze, and contextual and cued conditioned fear tasks. Neurobiol Learn Mem. 2008; 90:171-177.

19. Braden BB, Andrews MG, Acosta JI, et al. A comparison of progestins within three classes: Differential effects on learning and memory in the aging surgically menopausal rat. Behav Brain Res. 2017; 322(Pt B):258-268.

20. Johansson I-M, Birzniece V, Lindblad C, et al. Allopregnanolone inhibits learning in the Morris water maze. Brain Res. 2002; 934(2):125-131.

21. Nielsen SE, Segal SK, Worden IV, et al. Hormonal contraception use alters stress responses and emotional memory. Biol Psychol. 2013; 92(2):257-266.

22. Andreano J, Cahill L, Petersen N, et al. Oral contraceptive pill use is associated with localized decreases in cortical thickness. Human Brain mapping. Arch Dis Child. 2015; 62(4):362-369.

23. Kiernan JA, Rajakumar N. Barr's The Human Nervous System: An Anatomical Viewpoint. Philadelphia, Wolters Kluwer/ Lippincott Williams \& Wilkins. 2014.

24. Bruchey AK, Gonzalez-Lima F. Enhanced metabolic capacity of the frontal cerebral cortex after Pavlovian conditioning. Neurosci. 2008; 152(2):299-307.

25. Méndez-López M, Méndez M, López L, et al. Spatial working memory in young male and female rats: Involvement of different limbic system regions revealed by cytochrome oxidase activity. Neurosci Res. 2009; 65:28-34.

26. Devineni D, Skee D, Vaccaro N, et al. Pharmacokinetics and pharmacodynamics of a transdermal contraceptive patch and an oral contraceptive. J Clin Pharmacol. 2007; 47:497-509.

27. Bromley-Brits K, Deng Y, Song W. Morris water maze test for learning and memory deficits in Alzheimer's disease model mice. J Vis Exp. 2011; 53:2920.

28. Barnhart CD, Yang D, Lein PJ. Using the Morris water maze to assess spatial learning and memory in weanling mice. PLoS One. 2015; 10(4):e0124521.

29. Terry AV. Spatial navigation (water maze) tasks. In; Buccafusco JJ (Ed.). Methods of Behavior Analysis in Neuroscience. 2nd ed. Boca Raton (FL), CRC Press/Taylor \& Francis. 2009. 
30. Pletzer B, Kronbichler M, Aichhorn M, et al. Menstrual cycle and hormonal contraceptive use modulate human brain structure. Brain Res. 2010; 1348(12):55-62.

31. Griksiene R, Ruksenas O. Effects of hormonal contraceptives on mental rotation and verbal fluency. Psychoneuroendocrinol. 2011; 36(8):1239-1248.

32. Egan KR, Gleason CE. Longer duration of hormonal contraceptive use predicts better cognitive outcomes later in life. J Women Health (Larchmt). 2012; 21(12):1259-1266.

33. Martins DP, Semenoff TADV, Borges ÁH, et al. Chronic stress associated to contraceptives use on the progression of ligature-induced periodontitis in rats. Revista de Odontologia da UNESP. 2016; 45(5):290-296.

34. McEwen BS, Mirsky AE. Stress-induced remodelling of hippocampal CA3 pyramidal neurons. Brain Res. 2016; 1645: 50-54.

35. Watanabe Y, Gould E, McEwen BS. Stress induces atrophy of apical dendrites of hippocampal CA3 pyramidal neurons. Brain Res. 1992; 588(2):341-345.

36. Ekanem TB, Nwakanma AA, Eluwa MA, Ekong MB. Histological and immunohistochemical alterations of the amygdala of female Wistar rats administered oral ethinyl estradiol and levonogestrel combination. W J Biomed Res. 2020; 7(1):21-26.

37. Conrad CD, LeDoux JE, Magarinos AM, et al. Repeated restraint stress facilitates fear conditioning independently of causing hippocampal CA3 dendritic atrophy. Behav Neurosci. 1999; 113: 902-913.

38. Butterfield DA, Lange ML. Multifunctional roles of enolase in Alzheimer's disease brain: beyond altered glucose metabolism. J Neurochem. 2009; 111:915-933.

39. Ogundele OM, Madukwe J, Omotosho OA, et al. Immunohistochemical localization of neuron specific enolase and CD3 lymphocyte activation. J Med Sci. 2012; 12:92-98.
40. Hattori T, Takei N, Mizuno Y, et al. Neurotrophic and neuroprotective effects of neuron-specific enolase on cultured neurons from embryonic rat brain. Neurosci Res. 1995; 21(3):191-198.

41. Rosenstein JM. Developmental expression of neuron-specific enolase immunoreactivity and cytochrome oxidase activity in neocortical transplants. Exp Neurol. 1993; 124(2): 208-218.

42. Knapman A, Kaltwasser SF, Martins-de-Souza D, et al. Increased stress reactivity is associated with reduced hippocampal activity and neuronal integrity along with changes in energy metabolism. Eur J Neurosci. 2012; 35(3):412-422.

43. Oken BS, Chamine I, Wakeland W. A systems approach to stress, stressors and resilience in humans. Behav Brain Res. 2015; 282:144-154.

44. Paradiso K, Zhang J, Steinbach J. The $\mathrm{C}$ terminus of the human nicotinic alpha4beta2 receptor forms a binding site required for potentiation by an estrogenic steroid. J Neurosci. 2001; 21(17):6561-6568.

45. Nagele RG, Wegiel J, Venkataraman V, et al. Contribution of glial cells to the development of amyloid plaques in Alzheimer's disease. Neurobiol Aging. 2004: 25; 663-674.

46. Pekny M, Nilsson M. Astrocyte activation and reactive gliosis. Glia. 2005; 50(4):427-434.

47. Pekny M, Pekna A. Astrocyte intermediate filaments in CNS pathologies and regeneration. J Pathol. 2004; 204:428-437.

48. Nielsen SE, Ertman N, Lakhani YS, et al. Hormonal contraception usage is associated with altered memory for an emotional story. Neurobiol Learn Mem. 2011; 96(2):378.

49. Woolley C, Gould E, McEwen BS. Exposure to excess glucocorticoids alters dendritic morphology of adult hippocampal pyramidal neurons. Brain Res. 1990; 531:225-231.

50. Greenstein B, Greenstein A. Color Atlas of Neuroscience: Neuroanatomy and Neurophysiology. New York: Thieme. 2000. 